\title{
Unhealthy Mental States Are Positively Associated with Subjective Pain or Fatigue in Specific Body Sites among High School Teachers in Japan
}

\author{
Yuko Murakami, Hideki Tsumura, Rie Sato, Mari Fukuda, Hideyuki Kanda \\ Department of Environmental Medicine \& Public Health, Shimane University Faculty of Medicine, Shimane, Japan \\ Email: symk-605@nike.eonet.ne.jp
}

How to cite this paper: Murakami, Y., Tsumura, H., Sato, R., Fukuda, M. and Kanda, H. (2017) Unhealthy Mental States Are Positively Associated with Subjective Pain or Fatigue in Specific Body Sites among High School Teachers in Japan. Health, 9, 1313-1325.

https://doi.org/10.4236/health.2017.99095

Received: July 11, 2017

Accepted: September 15, 2017

Published: September 18, 2017

Copyright $\odot 2017$ by authors and Scientific Research Publishing Inc. This work is licensed under the Creative Commons Attribution International License (CC BY 4.0).

http://creativecommons.org/licenses/by/4.0/

\section{(c) (i) Open Access}

\begin{abstract}
Unhealthy mental states have become common among teachers recently. Subjective physical symptoms are more likely to appear more readily than mental symptoms. In this study, we revealed that the relationship between mental states and subjective physical fatigue or pain in seven body sites among 2449 teachers. The 12-item General Health Questionnaire (GHQ-12) was used to assess mental state. We defined a score of 4 points and over in the GHQ-12 score as unhealthy mental state. We also asked about the presence of subjective physical fatigue or pain in each seven body sites in the past one year. In the results, $30.3 \%$ unhealthy mental states were found in females and $22.9 \%$ in males. Most of subjective physical fatigue or pains were detected in neck and shoulder, eyes, head and low back. Using multivariate logistic regression, higher scores in the GHQ-12 were positively associated with presence of subjective fatigue or pain in head and eyes in both genders, in lower extremities among females, and in back, upper extremities among males. Our results suggest that physical symptoms in specific body sites with gender differences may be useful in the early identification of unhealthy mental states among teachers. We should have efforts to pay attention to mental states among high school teachers if their symptoms do not get better after taking physical care.
\end{abstract}

\section{Keywords}

The GHQ-12, Teacher, Mental State, Fatigue/Pain, Specific Body Site

\section{Introduction}

Recently, many teachers have shown unhealthy mental states due to lots of du- 
ties and relationships at work, which can be complicated [1] [2]. According to a survey by the Ministry of Education, Culture, Sports, Science and Technology, Japan (MEXT), 5009 full time teachers in Japanese public elementary, junior and senior high schools had administrative leave in 2015 due to mental illness [3]. The number of Japanese teachers who take administrative sick leave due to mental illness were showing an upwards trend. Over $60 \%$ of teachers who had administrative leave were suffered from mental illness [3]. Though without taking any administrative leave, approximately three times more teachers than those of general workers reported that they were "very fatigue" [4] [5]. The MEXT has taken measures for the teachers' mental state and recommendation to promote self-care as one aspect of preventive action.

Nakao et al. has reported that the emotional and cognitive symptoms of depression couldn't be obvious due to various types of complaints of physical troubles [6]. Patients are suffering from depression complain of physical signs, not depression, in outpatients treated in primary care [7]. There were few previous studies that examined the relationship between mental health and pain in various body regions among school teachers [8] [9]. Seibt et al. has found that unhealthy mental states were related to neck and back pain and headache especially in female teachers [8]. There is no epidemiological study on the relationship between mental states and physical signs in specific body sites among teachers in Japan.

The aim of our study was to clarify the relationship between mental state level and presence of the body fatigue or pain by seven body sites of 2449 teachers by gender in all junior and senior high schools in a local prefecture in Japan. The hypothesis was that subjective fatigue or pain in specific body sites were related to unhealthy mental states by both genders.

\section{Subjects and Methods}

\subsection{Study Subjects}

The subjects of this study were staff of all junior and senior high schools, including administers, in Shimane prefecture, Japan, 2015. The number of the teachers enrolled in 151 high schools (102 junior high schools (JHSs) and 47 senior high schools (SHSs)) was 3736 (1956 in JHSs and 1780 in SHSs) [10].

\subsection{Methods}

This study was a cross-sectional survey by anonymous self-reported questionnaire. The survey period was from August to the end of September 2015.

Request letters were sent to the school principal of the junior and senior high schools surveyed along with the questionnaire forms for all teachers in each school. All teachers in those schools were the targets of this questionnaire survey. Anonymous questions and envelopes were handed from their administrators to the teachers in participating schools and they were asked to complete during working hours. The complete questionnaires were sealed in the envelopes 
by the teachers themselves, collected by their administrators and returned to our research group unopened. This study was carried out as part of the JSPS KAKENHI Grant-in-Aid for Scientific Research (C) (Grant Number 15K08735). This study was approved by the institutional review board of Shimane University, Faculty of Medicine (No. 1863, approved 8 July 2015).

\subsection{Questionnaire}

\subsubsection{Demographics of Study Population}

We asked demographic data, sex, age category, school type (junior or senior high school), working duration and position.

\subsubsection{Mental State}

We used the 12-item General Health Questionnaire (GHQ-12) developed by Goldberg et al. as an indicator to evaluate state of mental state [11]. The GHQ-12 is the short version of the General Health Questionnaire. The GHQ-12 has been established as a measure for unhealthy mental state [12]. Previous studies confirmed its validation and reliability properties [12]. We evaluated teachers' mental status by the GHQ-12 scores as follows [11]. The questionnaire consists of 12 items to assess the severity of a mental problem. There are four answer choices in each item, including 2 positive answers and 2 negative answers. For the GHQ-12 we used the 0-0-1-1 scoring method. The GHQ scoring has been widely adopted in previous studies [13] [14] [15]. We gave 0 point for the positive answers and 1 point for the negative answers. All items were added to obtain the total score. The 12 items total score was from 0 point to 12 points. A higher score indicated unhealthier mental state. Based on previous studies, we defined 4 points in the GHQ-12 as unhealthy mental group [14] [16].

\subsubsection{Subjective Physical Pain or Fatigue in Body Sites}

We asked about the presence of physical fatigue or pain in the past one year period in seven body sites-head, eyes, neck and/or shoulders, arms and/or hands and fingers, back, lower back, lower extremities-based on the items surveyed by the Ministry of Health, Labor and Welfare, Japan (MHLW) in 2008 [17]. Subjects were given a list of subjective physical pain or fatigue in body sites and selected all the body sites they had felt pain or fatigue.

\subsection{Statistical Analysis}

Statistical analysis was conducted separately by gender. Chi-squaretest was used to compare the frequency of the characteristic on subjects between mentally healthy group and mentally unhealthy group: age group, sex, school type, working period and position. Since there were few female subjects in the position of school principal or vice-principal or head teacher, Fisher's exact test was used for female working positions. Then, Chi-squaretest was used to compare the frequency of subjective pain or fatigue in each body site between mentally healthy group and mentally unhealthy group. 
Multiple logistic regression analyses were used to assess the contribution of each independent variable to the unhealthy mental state. We examined two multivariable logistic regression models; the model 1 was adjusted by each one body site and characteristic items that showed a significant difference, and the model 2 was adjusted by all seven body sites and characteristic items that showed a significant difference.

The statistics software EZR version 1.33 was used for the analyses [18]. All probability values were two-tailed and at a $5 \%$ level of significance.

\section{Results}

We received responses from 123 high schools (school response rate 82.6\%; 76 JHSs and 47 SHSs). The number of questionnaires collected by individual teachers was 2484 (individual response rate: 66.5\%) for 3736 subjects. Of 2484 responses, 35 responses did not have the complete data necessary for analysis. Thus, 2449 eligible responses (of which 860 females and 1589 males) participated in this study.

Table 1 shows the characteristics of mentally healthy/unhealthy groups by gender. Two hundred sixty one females (30.3\% in females) and 364 males $(22.9 \%$ in males) were in mentally unhealthy group. The prevalence of mentally unhealthy group was higher in females who have worked for less than 5 years

Table 1. Characteristics of mentally healthy and unhealthy groups by gender.

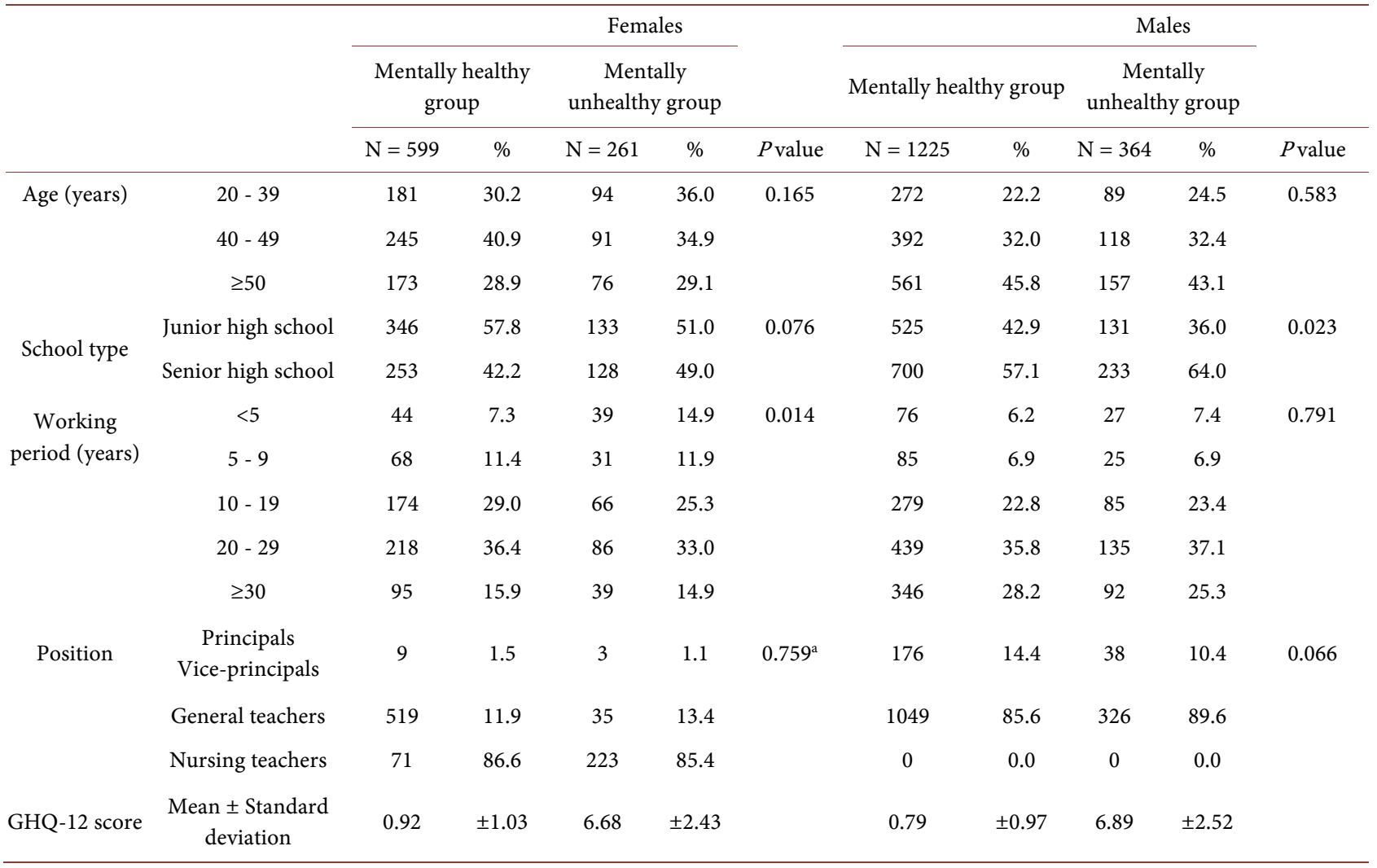

$P$ value was calculated by $\chi^{2}$ test. ${ }^{\text {aP }}$ value was calculated by Fisher's exact test. 
compared to that of mentally healthy group ( $14.9 \%$ in mentally unhealthy group vs. $7.3 \%$ in mentally healthy group). In males, there were more teachers who were working at SHSs in mentally unhealthy group than in mentally healthy group with statistical significance $(64.0 \%$ in mentally unhealthy group vs. $57.1 \%$ in mentally healthy group). It did not reach a statistical difference between their mental states and age groups, as well as position, in both genders.

Table 2 shows the comparisons of prevalence on subjective fatigue or pain in each body site by mental health state and gender. In both male and female, the prevalence of subjective fatigue or pain in every body site was significantly higher in mentally unhealthy group. The prevalence of subjective fatigue or pains of neck-shoulder, subsequently eyes, were the significantly highest and more than half teachers in the mentally unhealthy group claimed them.

Table 3 shows the odds ratios and 95\% confidence intervals of mentally unhealthy states as determined using multivariate logistic regression by gender. In females, adjusting working period and one item for each body site on the model 1, higher score for the GHQ-12 was significantly related with subjective fatigue or pain in all each body site. Adjusting the working period and all body sites on the model 2 in females, mentally unhealthy states were statistically positively associated with the presence of subjective fatigue or pain in eyes, low extremities,

Table 2. Comparisons of prevalence on subjective fatigue or pain in each body site by mentally healthy states and gender.

\begin{tabular}{|c|c|c|c|c|c|}
\hline \multirow{3}{*}{$\begin{array}{c}\text { Presence of fatigue or pain } \\
\text { in body site }\end{array}$} & \multicolumn{5}{|c|}{ Females } \\
\hline & \multicolumn{2}{|c|}{ Mentally healthy group } & \multicolumn{3}{|c|}{ Mentally unhealthy group } \\
\hline & $\mathrm{N}=599$ & $\%$ & $\mathrm{~N}=261$ & $\%$ & $P$ value \\
\hline Neck-Shoulder & 430 & 71.8 & 211 & 80.8 & 0.007 \\
\hline Eyes & 306 & 51.1 & 174 & 66.7 & $<0.001$ \\
\hline Head & 249 & 41.6 & 147 & 56.3 & $<0.001$ \\
\hline Low back & 175 & 29.2 & 96 & 36.8 & 0.034 \\
\hline Lower extremities & 114 & 19.0 & 74 & 28.4 & 0.003 \\
\hline Upper back & 92 & 15.4 & 64 & 24.5 & 0.002 \\
\hline \multirow[t]{2}{*}{ Upper extremities } & 76 & 12.7 & 48 & 18.4 & 0.037 \\
\hline & \multicolumn{5}{|c|}{ Males } \\
\hline \multirow{2}{*}{$\begin{array}{l}\text { Presence of fatigue or pain } \\
\text { in body site }\end{array}$} & \multicolumn{2}{|c|}{ Mentally healthy group } & \multicolumn{2}{|c|}{ Mentally unhealthy group } & \\
\hline & $\mathrm{N}=1225$ & $\%$ & $\mathrm{~N}=364$ & $\%$ & $P$ value \\
\hline Neck-Shoulder & 643 & 52.5 & 241 & 66.2 & $<0.001$ \\
\hline Eyes & 512 & 41.8 & 215 & 59.1 & $<0.001$ \\
\hline Low back & 438 & 35.8 & 153 & 42.0 & 0.035 \\
\hline Head & 251 & 20.5 & 121 & 33.2 & $<0.001$ \\
\hline Upper back & 161 & 13.1 & 85 & 23.4 & $<0.001$ \\
\hline Lower extremities & 196 & 16.0 & 77 & 21.2 & 0.027 \\
\hline Upper extremities & 138 & 11.3 & 75 & 20.6 & $<0.001$ \\
\hline
\end{tabular}

P value was calculated by $\chi^{2}$ test. 
Table 3. Odds ratios and 95\% confidence interval for mentally unhealthy group by gender.

\begin{tabular}{|c|c|c|c|c|c|c|c|c|}
\hline & \multicolumn{8}{|c|}{ Female $(\mathrm{N}=860)$} \\
\hline & \multicolumn{4}{|c|}{ Working period-adjusted ${ }^{a}$} & \multicolumn{4}{|c|}{ Multivariable-adjusted $^{\mathrm{b}}$} \\
\hline & Odds ratio & \multicolumn{2}{|c|}{ 95\% Confidence intervals } & $P$ value & Odds ratio & \multicolumn{2}{|c|}{ 95\% Confidence intervals } & $P$ value \\
\hline Body sites & & Minimum & Maximum & & & Minimum & Maximum & \\
\hline Eyes & 1.950 & 1.440 & 2.650 & $<0.001$ & 1.560 & 1.130 & 2.160 & 0.007 \\
\hline Lower extremities & 1.740 & 1.240 & 2.440 & 0.001 & 1.440 & 1.000 & 2.060 & 0.048 \\
\hline Head & 1.780 & 1.320 & 2.390 & $<0.001$ & 1.400 & 1.020 & 1.920 & 0.037 \\
\hline Upper back & 1.810 & 1.260 & 2.600 & 0.001 & 1.300 & 0.873 & 1.940 & 0.195 \\
\hline Neck-shoulder & 1.700 & 1.190 & 2.430 & 0.004 & 1.250 & 0.852 & 1.840 & 0.253 \\
\hline Upper extremities & 1.630 & 1.090 & 2.430 & 0.017 & 1.200 & 0.786 & 1.840 & 0.394 \\
\hline \multirow[t]{4}{*}{ Low back } & 1.430 & 1.050 & 1.950 & 0.023 & 1.010 & 0.718 & 1.430 & 0.942 \\
\hline & \multicolumn{8}{|c|}{ Male $(\mathrm{N}=1589)$} \\
\hline & \multicolumn{4}{|c|}{ School type-adjusted ${ }^{\mathrm{c}}$} & \multicolumn{4}{|c|}{ Multivariable-adjusted $^{\mathrm{d}}$} \\
\hline & Odds ratio & \multicolumn{2}{|c|}{ 95\% Confidence intervals } & $P$ value & Odds ratio & \multicolumn{2}{|c|}{ 95\% Confidence intervals } & $P$ value \\
\hline Body sites & & Minimum & Maximum & & & Minimum & Maximum & \\
\hline Eye & 2.020 & 1.590 & 2.560 & $<0.001$ & 1.550 & 1.190 & 2.020 & 0.001 \\
\hline Upper extremities & 2.030 & 1.490 & 2.770 & $<0.001$ & 1.500 & 1.060 & 2.120 & 0.022 \\
\hline Head & 1.950 & 1.500 & 2.520 & $<0.001$ & 1.460 & 1.100 & 1.930 & 0.009 \\
\hline Upper back & 2.010 & 1.500 & 2.710 & $<0.001$ & 1.460 & 1.040 & 2.040 & 0.027 \\
\hline Neck-shoulder & 1.790 & 1.400 & 2.280 & $<0.001$ & 1.270 & 0.965 & 1.670 & 0.089 \\
\hline Low back & 1.320 & 1.040 & 1.680 & 0.022 & 0.952 & 0.725 & 1.250 & 0.723 \\
\hline Lower extremities & 1.420 & 1.060 & 1.910 & 0.019 & 0.909 & 0.642 & 1.290 & 0.591 \\
\hline
\end{tabular}

aadjusted by work period. badjusted by fatigue or pain of each body site of work period, lower extremities, neck-shoulder, eyes, upper back, upper extremities, low back and head. cadjusted by junior or senior high school. ${ }^{\mathrm{d}}$ adjusted by fatigue or pain of each body site of junior or senior high school, lower extremities, neck-shoulder, eyes, upper back, upper extremities, low back and head.

and head. In males, adjusting school type and one item for each body site on the model 1, like females, higher score for the GHQ-12 was significantly related to subjective fatigue or pain in all each body site. Adjusting by school type and all body sites on the model 2 , mentally unhealthy state was statistically positively associated with the presence of subjective fatigue or pain in not only eyes but also upper extremities and upper back.

\section{Discussion}

We clarified that there was a relationship between mentally unhealthy states and subjective fatigue or pain in several body sites, especially eyes and head in both genders. We also found that mentally unhealthy group had gender differences in body site where they felt subjective fatigue or pain; low extremities in females and upper extremities and upper back in males. This study is the first epidemiological study in Japan to focus on the relationships between mental states and fatigue or pain in body sites by gender among high school teachers.

Japanese junior high school teachers have the longest work hours per a week 
in the world, which is 54 hours (world average of 38.3 hours) [19]. They spend long time on their duties especially out of classes, such as general office work and extracurricular activities. Previous study has been reported that teacher's daily working hours in Japan is approximately five hours longer than that in Finland, and 2.5 hours longer than that in the UK [20]. It has also indicated that Japanese teacher' break hours are less than half, and sleep hours are one $80 \mathrm{mi}$ nutes shorter than those in other countries [20].

The MEXT has reported that the backgrounds for teachers' mental illness were include-increasing work volume and quality demanded [21]. There were 5009 teachers who took administrative sick leave due to mental illness in 2015 [3]. It has stayed around 5000 since 2007 and has continuously been slightly decreasing for the past two years [2] [3]. According to a survey conducted by the MEXT, about $60 \%$ of the teachers taking sick leave were reported to take it because of mental illness [3]. Further, the number of the teachers who had mental illness was up to approximately $60 \%$ of all teachers' resignees. Additionally, there were teachers who complaints "very fatigue" was three times more than general workers [4] [5]. In this study, we examined the relationships between mental health and body fatigue or pain among teachers.

This study clarified a relationship between unhealthy mental state and subjective fatigue or pain in three body sites in females and in four body sites in males. The previous studies on mental illness in which GHQ-12 was used has reported the relationship between mental illness and subjective fatigue or pain in each body site; head, neck, shoulders, back, and lower back, respectively [8] [22] [23]. Seibt et al. has shown that $78 \%$ of mentally unhealthy female teachers felt pain in the neck and back and 58\% had headaches [8]. Research in the UK has reported a relationship between lower mental states and shoulder pain among workers of five differing job types, including mail carriers and cashiers [23]. Several studies have reported a relationship between mentally unhealthy states defined by the GHQ-12 and lower-back pain [22] [23]. These previous studies asked subjective physical fatigue or pain in body sites within almost 2 weeks before the survey. We asked the questions on pain or fatigue within the past one year. Our results may show the effect to unhealthy mental states by long period exposure to subjective pain or fatigue. We found a relationship between unhealthy mental states and all each pain or fatigue in seven sites of the body by the model 1 as a simple statistical model. Our results can also reveal the relationship between unhealthy mental states and physical symptoms over long periods of time up to one year. Moreover, using the model 2 as a multivariate model, this study clarified a relationship between unhealthy mental states and physical symptoms in eyes and head in both genders; and in lower extremities in females, and in upper extremities and upper back in males. Our results suggest that the presence of long-term pain, particularly pain in a specific site of the body, may be related between particularly pain in a specific site of the body and unhealthy mental states. There are several studies that chronic pain is related to depression. There are few studies that clarified whether pain is first or depression is ahead. The causal relationship 
is not clear. However, the some previous studies have revealed that a relationship between long term pain and unhealthy mental state [24] [25].

We found a relationship between unhealthy mental states and headaches in both genders. The previous studies have shown positive relationships between headaches and mental illness in females [8] [26]. Another study has shown that those who have tension-type headache tend to have anxiety and depression than those who do not have [27]. Jaradat et al. have described that there was a significantly positive relationship between harsh work stress and tension-type headaches among nurses in both genders [28]. Not only tension-type headaches but also migraines are related to depression and anxiety [29] [30]. There also have a report that drug induced headaches are affiliated with anxiety [30]. Another study has been found that $43 \%$ in patients with chronic cluster headache had higher depression scores than those who did not [31]. Moreover, there have been reports patients with anxiety and depression were frequently observed to have chronic daily headaches [32]. Our finding was supported by many previous studies to have proved that various types of headaches may affect mental states.

Our study also indicated that fatigue or pain of eyes was related to unhealthy mental state in both genders. Dehghan $\mathrm{H}$ et al. have found a positive association between anxiety score and eye fatigue [33]. Araki et al. reported a significantly positive relationship between eye discomfort and depression in only females [26]. Another study has been shown that dry eye was observed almost twice as often in individuals with mental illness, such as depression and PTSD, as those without [34]. On the other hand, Zhaoji et al. have described that there was no relationship between eye strain and the GHQ-12 score in females who use VDT [22]. Thus, there had been no consensus between eye symptoms and mental states. We identified the relationship between eye symptoms and unhealthy mental status by a large-scale epidemiological survey.

Moreover, our findings showed gender differences between unhealthy mental states and fatigue or pain in specific body sites except eyes and head. We found a relationship between unhealthy mental states in the GHQ-12 and fatigue or pain of lower extremities in females, or both upper extremities and back in males. There have been few papers indicating gender differences between mental states and physical symptoms in simultaneous surveys of both genders. Ceballos' survey has reported that the prevalence of teachers with musculoskeletal pain was over $70 \%$ [35]. Darwish et al. confirmed that $40 \%$ of female teachers had foot pain [36]. Teachers stand on most of the class hours and write letters on a big blackboard in their daily lives. Teachers often have the behaviors that stand for about 6 hours a day in total. Therefore, it is inferred that fatigue or pain occurs on foot. It appears that some of the reasons can be explained by the above previous studies. However, we have had not the detail data for comparison between teachers and non-teachers. Previous studies have been reported that women have higher prevalence of knee pain than men [37] [38]. One of the reasons why women have higher prevalence of knee pain may be due to structural differences in knee from male, including cartilage thickness, volume and joint surface area 
[39] [40]. Fatigue or pain in the lower extremities may also occur due to hallux valgus which female tend to have more than male [41]. Mizuno et al. reported that damage to the digiti minimi muscle (ADMM) nerve and causes atrophy, which is often seen in females, and that this may be one factor for pain in the heel ultimately [42]. Kawakami et al. have shown that almost $90 \%$ of females (15\% of males) are suffering from lower extremities edema [43]. Zöllner et al. argue that women often wear high-heeled shoes and this may cause fatigue and pain in lower extremities [44]. Based the findings above, there has been a report that females complain foot fatigue or pain more often than males. In contrast, it has been reported that males are three times more likely to break their metacarpal bones than females [38]. This result suggests that men tend to have hand injuries than women. We could not find any researches comparing between male with female on back pain among high school teachers. In previous research, the subjective prevalence on back pain in females was approximately double than that in males [36]. This was different from our results. Previous research examined pain within a week, whereas we asked on fatigue/pain within a year. This difference in time span would be one factor that led to different results. Furthermore, according to the previous research on computer users, unhealthy mental states were related to back pain [45]. According to the report, men have more back pain than women do because men were generally taller than women and operated computers with unnatural position [45]. Although we have not asked the details that how long teachers stand, sit, use computers, teachers spend much time using personal computers, such as grading work, documentation, reports, contacting parents, and creating documents. It has been reported that teachers often complain of musculoskeletal pain [35]. Pain and fatigue are believed to occur from using the computer in the same or in a strained position. Therefore, male teachers may often complain of fatigue or pain in the back. Our results on gender differences may probably reflect some of known factors in previous studies.

The strengths of this study are as follows. First, this was a large-scale epidemiological survey that targeted all teachers of all high schools in a single prefecture, Japan. Then, we investigated the distribution of fatigue or pain sites from each seven body, considering their mental status.

There are some limitations in this study. First, our study uses a cross-sectional design, which does not prove a causal relation. Second, there were limits to the questionnaire form used in this study. We asked only subjective presences of fatigue or pain in the body sites so we could not distinguish fatigue with pain in the body regions. Third, we have not got detailed data how long teachers stand, sit, and use computers; we could not make exact comparisons between the teachers and the general people.

\section{Conclusion}

In conclusion, we clarified a relationship between unhealthy mental state and fa- 
tigue or pain in various body sites among high school teachers. When someone complains physical symptoms such as headache or eye fatigue, first we should encourage them to visit a clinic for physical check-up. If the symptoms do not get better, we have to think the possibility of unhealthy mental states and we suggest him or her to take counseling or to visit a psychological professional. It is important to improve unhealthy mental states at early stages. Our results indicate that fatigue or pain in specific body site by gender may help to detect unhealthy mental states at earlier stage among high school teachers.

\section{Acknowledgements}

We are grateful to Ms. Toshimi Yoneyama and Ms. Izumi Okui for the collection of the data. This study was supported by a JSPS KAKENHI Grant-in-Aid for Scientific Research (C) (Grant Number 15K08735).

\section{References}

[1] Tagami, F., Yamamoto, J. and Tanaka, T. (2004)Teachers' Mental Health: A Review (Overviews on Current Research Topics). The Annual Report of Educational Psychology in Japan, 43, 135-144. https://doi.org/10.5926/arepj1962.43.0 135

[2] Okuno, Y. (2013) Teacher's Mental Health. Bulletin of Center for Clinical Psychology Kinki University, 6, 33-41. (In Japanese)

[3] Ministry of Education, Culture Sports, Science and Technology (2016) About the Investigation on the Personnel Administration Situation of the Public-School Faculty Staff in 2015. (In Japanese) http://www.mext.go.jp/a menu/shotou/jinji/1380718.htm

[4] Ministry of Education, Culture, Sports, Science and Technology (2012) Status of Mental Health of Teachers. (In Japanese)

http://www.mext.go.jp/b menu/shingi/chousa/shotou/088/shiryo/ icsFiles/afieldfi le/2012/02/24/1316629 001.pdf

[5] Ministry of Health, Labor and Welfare. (2002) Status of Mental Health of Workers. (In Japanese) http://www.mhlw.go.jp/toukei/itiran/roudou/saigai/anzen/kenkou02/r1.html

[6] Nakao, M. and Yano, E. (2006) Somatic Symptoms for Predicting Depression: One-Year Follow-Up Study in Annual Health Examinations. Psychiatry and Clinical Neurosciences, 60, 219-225. https://doi.org/10.1111/j.1440-1819.2006.01489.x

[7] Tsuboi, K. (2017) Differential Diagnosis of Depressive State. (In Japanese) http://jams.med.or.jp/symposium/full/129040.pdf

[8] Seibt, R., Spitzer, S., Druschke, D., Scheuch, K. and Hinz, A. (2013) Predictors of Mental Health in Female Teachers. International Journal of Occupational Medical and Environmental Health, 26, 856-869. https://doi.org/10.2478/s13382-013-0161-8

[9] Zamri, E., Moy, F. and Hoe, V. (2017) Association of Psychological Distress and Work Psychosocial Factors with Self-Reported Musculoskeletal Pain among Secondary School Teachers in Malaysia. PLoS One, 12, Article ID: e0172195. https://doi.org/10.1371/journal.pone.0172195

[10] Shimane Prefectural Goverment Policy Planning Bureau Statistical Survey Division (2016) School Basic Survey Result Report for 2015 (Shimane Prefecture). (In Japanese) http://pref.shimane-toukei.jp/index.php?view=18652 
[11] Goldberg, D.P., Gater, R., Sartorius, N., Ustun, T.B., Piccinelli, M., Gureje, O. andRutter, C. (1997) The Validity of Two Versions of the GHQ in the WHO Study of Mental Illness in General Health Care. Psychological Medicine, 27, 191-197. https://doi.org/10.1017/S0033291796004242

[12] Doi, Y. and Minowa, M. (2001) Factor Structure of the 12-Item General Health Questionnaire in the Japanese General Adult Population. Psychiatry and Clinical Neurosciences, 57, 379-383. https://doi.org/10.1046/j.1440-1819.2003.01135.x

[13] Godinho, E.L., Farias, L.C., Aguiar, J.C., Martelli-Júnior, H., Bonan, P.R., Ferreira, R.C., et al. (2011) No Association between Periodontal Disease and GHQ-12 in a Brazilian Police Population. Medicina Oral Patologia Oral y Cirugia Bucal, 16, 857-863. https://doi.org/10.4317/medoral.17173

[14] Saito, N., Yamamoto, T. and Kitaike, T. (2016) Work Environments for Healthy and Motivated Public Health Nurses. Nihon Koshu Eisei Zasshi, 63, 397-408. (In Japanese)

[15] Matsuzaki, I., Sagara, T., Ohshita, Y., Nagase, H., Ogino, K., Eboshida, A., et al. (2007) Psychological Factors Including Sense of Coherence and Some Lifestyles Are Related to General Health Questionnaire-12 (GHQ-12) in Elderly Workers in Japan. Environ Health Preventive Medicine, 12, 71-77. https://doi.org/10.1007/BF02898152

[16] Honda, S., Shibata, Y. and Nakane, Y. (2003) Screening for Psychiatric Disorders Using the 12-Item General Health Questionnaire. Journal of Health and Welfare, 48, 5-10. (In Japanese)

[17] Ministry of Health, Labor and Welfare (2009) Survey on Actual State of Innovation and Labor in 2008. (In Japanese)

http://www.mhlw.go.jp/toukei/itiran/roudou/saigai/anzen/08/02.html

[18] Kanda, Y. (2013) Investigation of the Freely Available Easy-to-Use Software "EZR" for Medical Statistics. Bone Marrow Transplantation, 48, 452-458. https://doi.org/10.1038/bmt.2012.244

[19] National Institute for Educational Policy Research (2014) Teaching and Learning International Survey "Summary of the Results of the 2013 Survey". (In Japanese) http://www.nier.go.jp/kenkyukikaku/talis/imgs/talis2013 summary.pdf

[20] National Institute of Education and Culture (2007) Lesson Preparation and Time to Face Children. (In Japanese) http://www.k-soken.gr.jp/files/lib/2/38/201605241912527510.pdf

[21] Ministry of Education, Culture, Sports, Science and Technology (2013) Ministry of Education, Culture, Sports, Science and Technology. States of Mental Health of Teachers. (In Japanese) http://www.mext.go.jp/component/b menu/shingi/toushin/ icsFiles/afieldfile/201 3/03/29/1332655 03.pdf

[22] Zhaojia, Y.E., Honda, S., Abe, Y., Kusano, Y., Takamura, N., Imamura, Y., et al. (2007) Influence of Work Duration or Physical Symptoms on Mental Health among Japanese Visual Display Terminal Users. Industrial Health, 45, 328-333. https://doi.org/10.2486/indhealth.45.328

[23] Pope, D.P., Silman, A.J., Cherry, N.M., Pritchard, C. and Macfarlane, J. (2001) Association of Occupational Physical Demands and Psychosocial Working Environment with Disabling Shoulder Pain. Annals Rheumatic Diseases, 60, 852-858.

[24] Ohayon, M.M. and Schatzberg, A.F. (2003) Using Chronic Pain to Predict Depressive Morbidity in the General Population. Arch Gen Psychiatry, 60, 39-47. https://doi.org/10.1001/archpsyc.60.1.39 
[25] Gureje, O., Simon, G.E. and Michael, V.K. (2001) A Cross-National Study of the Course of Persistent Pain in Primary Care. Pain, 92, 195-200.

[26] Araki, Y., Muto, T. and Asakura, T. (1999) Psychosomatic Symptoms of Japanese Working Women and Their Need for Stress Management. Industrial Health, 37, 253-262. https://doi.org/10.2486/indhealth.37.253

[27] Song, T.J., Cho, S.J., Kim, W.J., Yang, K.I., Yun, C.H. and Chu, M.K. (2016) Anxiety and Depression in Tension-Type Headache: A Population-Based Study. PLoS ONE, 11, e0165316. https://doi.org/10.1371/journal.pone.0165316

[28] Jaradat, Y., Nijem, K., Lien, L., Stigum, H., Bjertness, E. and Bast-Pettersen, R. (2016) Psychosomatic Symptoms and Stressful Working Conditions among Palestinian Nurses: A Cross-Sectional Study. Contemporary Nurse, 52, 381-397. https://doi.org/10.1080/10376178.2016.1188018

[29] Peres, M.F.P., Mercante, J.P.P., Tobo, P.R., Kamei, H. and Bigal, M.E. (2017) Anxiety and Depression Symptoms and Migraine: A Symptom-Based Approach Research. The Journal of Headache and Pain Headache Pain, 18, 37. https://doi.org/10.1186/s10194-017-0742-1

[30] Lampl, C., Thomas, H., Tassorelli, C., Katsarava, Z., Lainez, J.M., Lanteri-Minet, M., et al. (2016) Headache, Depression and Anxiety: Associations in the Eurolight Project. The Journal of Headache and Pain, 17, 59. https://doi.org/10.1186/s10194-016-0649-2

[31] Donnet, A., Lanteri-Minet, M., Guegan-Massardier, E., Mick, G., Fabre, N., Geraud, G., et al. (2007) Chronic Cluster Headache: A French Clinical Descriptive Study. Journal of Neurology, Neurosurgery and Psychiatry, 78, 1354-1358. https://doi.org/10.1136/jnnp.2006.112037

[32] Láinez, M.J. (2005) Chronic Headaches: From Research to Clinical Practice. The Journal of Headache and Pain, 6, 175-178. https://doi.org/10.1007/s10194-005-0177-y

[33] Dehghan, H., Azmoon, H., Souri, S. and Akbari, J. (2014) The Effects of State Anxiety and Thermal Comfort on Sleep Quality and Eye Fatigue in Shift Work Nurses. Journal of Education and Health Promotion, 3, 72.

[34] Galor, A.L., Feuer, W., Lee, D.J., Florez, H., Carter, D., Pouyeh, B., et al. (2011) Prevalence and Risk Factors of Dry Eye Syndrome in a United States Veterans Affairs Population. American Journal of Ophthalmology, 152, 377-384.

[35] Ceballos, A.G.C. and Santos, G.B. (2015) Factors Associated with Musculoskeletal Pain among Teachers: Sociodemographics Aspects, General Health and Well-Being at Work. Revista Brasileira de Epidemiologia, 18, 702-715. https://doi.org/10.1590/1980-5497201500030015

[36] Darwish, M.A. and Al-Zuhair, S.Z. (2013) Musculoskeletal Pain Disorders among Secondary School Saudi Female Teachers. Pain Research and Treatment, 2013, Article ID: 878570.

[37] Glass, N., Segal, N.A., Sluka, K.A., Torner, J.C., Nevitt, M.C., Felson, D.T., et al. (2014) Examining Sex Differences in Knee Pain: The Multicenter Osteoarthritis Study. Osteoarthritis Cartilage, 22, 1100-1106.

[38] Wolf, J.M., Lisa, C., Heest, A.E., O’Connor, M. and Ladd, A.L. (2015) Male and Female Differences in Musculoskeletal Disease. Journal of the American Academy of Orthopaedic Surgeons, 23, 339-347. https://doi.org/10.5435/JAAOS-D-14-00020

[39] Otterness, I.G. and Eckstein, F. (2007) Women Have Thinner Cartilage and Smaller Joint Surfaces than Men after Adjustment for Body Height and Weight. Osteoarthritis and Cartilage, Osteoarthritis Research Society, 15, 666-672. 
[40] Cicuttini, F., Forbes, A., Morris, K., Darling, S., Bailey, M. and Stuckey, S. (1999) Gender Differences in Knee Cartilage Volume as Measured by Magnetic Resonance Imaging. Osteoarthritis and Cartilage, Osteoarthritis Research Society, 7, 265-271. https://doi.org/10.1053/joca.1998.0200

[41] Nix, S., Smith, M. and Vicenzino, B. (2010) Prevalence of Hallux Valgus in the General Population: A Systematic Review and Meta-Analysis. Journal of Foot and Ankle Research, 3, 21. https://doi.org/10.1186/1757-1146-3-21

[42] Mizuno, D., Naito, M., Hayashi, S., Ohmichi, Y., Ohmichi, M. and Nakano, T. (2015) Sex Differences in the Branching Position of the Nerve to the Abductor Digiti Minimi Muscle: An Anatomical Study of Cadavers. Journal of Foot and Ankle Research, 8, 22. https://doi.org/10.1186/s13047-015-0077-6

[43] Kawakami, K., Kawamoto, T. and Yamazaki, N. (2007) Design of an Office Chair to Reduce Swelling in Female VDT Workers. Japan Ergonomics Society, 43, 252-260. https://doi.org/10.5100/jje.43.252

[44] Zöllner, A.M., Pok, J.M., McWalter, E.J., Gold, G.E. and Kuhl, E. (2015) On High Heels and Short Muscles: A Multiscale Model for Sarcomere Loss in the Gastrocnemius Muscle. Journal of Theoretical Biology, 365, 301-310.

[45] Cho, C.Y., Hwang, Y.S. and Cherng, R.J. (2012) Musculoskeletal Symptoms and Associated Risk Factors among Office Workers with Workload Computer Use. Journal of Manipulative and Physiological Therapeutics, 35, 534-540.

\section{Submit or recommend next manuscript to SCIRP and we will provide best service for you:}

Accepting pre-submission inquiries through Email, Facebook, LinkedIn, Twitter, etc. A wide selection of journals (inclusive of 9 subjects, more than 200 journals) Providing 24-hour high-quality service

User-friendly online submission system

Fair and swift peer-review system

Efficient typesetting and proofreading procedure

Display of the result of downloads and visits, as well as the number of cited articles

Maximum dissemination of your research work

Submit your manuscript at: http://papersubmission.scirp.org/

Or contact health@scirp.org 